\title{
Integrating Renewable Energy Contracts and Wholesale Dynamic Pricing to Serve Aggregate Flexible Loads
}

\author{
Anthony Papavasiliou, Member, IEEE, and Shmuel S. Oren, Fellow, IEEE
}

\begin{abstract}
In this paper we describe the use of wholesale dynamic pricing for coordinating the demand schedule of a load aggregator which is responsible for matching the consumption of a large population of flexible loads, like EV batteries, with renewable energy resources. We formulate a stochastic optimal control problem that describes the decision problem faced by the aggregator, and we use a dynamic programming algorithm to solve the problem. We control the computational complexity of the algorithm by modeling correlated uncertain variables with a recombinant lattice. We then compare the relative performance of the optimal policy to alternative charging policies, and investigate the sensitivity of our results with respect to the cost of unserved energy and the degree to which the aggregator can respond to dynamic pricing.
\end{abstract}

Index Terms-Dynamic pricing, renewable energy, dynamic programming.

\section{INTRODUCTION}

Dynamic pricing has the potential to materialize the potential benefits of demand side flexibility on power system operations. These benefits are especially pronounced in systems where renewable resource integration is increasing rapidly, due to the fact that renewable energy supply varies unpredictable and beyond the control of the system operator. The impacts of renewable energy integration on reserve requirements and operational costs are analyzed thoroughly in numerous renewable energy integration reports [1], [2], [3], [4]. Demand side flexibility can alleviate many of the operational problems that arise from renewable energy integration through dynamic pricing [5] or the use of loads for providing ancillary services [6], [7], [8].

In this paper we study the effects of wholesale dynamic pricing on the operations of a load aggregator, rather than the effects of broadcasting pricing signals directly to retail customers. We propose a contract which couples the supply of renewable energy suppliers to large groups of deferrable loads. The coupling is coordinated by an aggregator which uses renewable energy assets to cover the majority of flexible demand, and resorts to spot market procurements to a limited extent, in order to mitigate the risk of unreliable renewable energy supply.

In our proposed model, renewable suppliers dedicate their supply to large groups of deferrable loads. The consumption of the flexible loads is managed by an aggregator which

A. Papavasiliou and S. S. Oren are with the Department of Industrial Engineering and Operations Research, UC Berkeley, Berkeley, CA, 94709 USA (e-mail: tonypap@ berkeley.edu; oren@ieor.berkeley.edu.

978-1-4577-1002-5/11/\$26.00 C2011 IEEE also has the option of resorting to the real time electricity market in order to hedge against the uncertainty of renewable energy supply. In the contracts which we consider, consumers specify their flexible consumption tasks as requests for a certain amount of energy within a certain deadline. The task of the aggregator is to fulfill these requests primarily from renewable resources. The aggregator then operates the contracted renewable energy assets and deferrable loads independently form the entire system, and only interacts with the system operator through the real time market, thereby making renewable energy sources appear "behind the meter" from the point of view of the system operator. In order for this contract to reduce reserve requirements associated with the large scale integration of renewable supply, we assume that the residual risk of renewable supply shortages is assumed by the aggregator. For this reason, in our model we impose a limit on the degree to which the aggregator can participate in the spot market, and we impose a penalty on the aggregator for failing to fulfill demand. In effect, the coupling contract transfers the risk of renewable energy fluctuations from the system operator to the aggregator. The coupling contract which we describe closely resembles dynamic scheduling, as described in [9].

The coupling contract which we study possesses various attractive features. Many flexible power consumption tasks can be naturally described in terms of deadlines for a given amount of energy demand. Such tasks include the charging of electric vehicles, water pumping, plant cultivation in greenhouses and requests for computational tasks in server farms. Moreover, due to the fact that aggregate renewable supply tends to be more predictable over a given time horizon rather than at any given moment in time, there is a natural complementarity of coupling renewable supply with consumers which can specify their demand over a given time horizon. Nevertheless, the contract assumes that the aggregator directly controls customers' loads, which may be undesirable by customers. In addition, matching specific collections of loads with specific renewable resources results in losses of trading efficiency and also reduced benefits from geographical diversification of renewable energy supply. Although providing dynamic price signals directly to retail customers is the most effective solution for capturing the potential benefits of demand flexibility, policy makers are reluctant to expose retail consumers to the volatility of electricity prices. In our future research we wish to compare the benefits of exposing an aggregator to wholesale dynamic pricing versus the benefits of directly exposing retail customers to dynamic pricing. 


\section{Problem Formulation}

We consider the task of an aggregator which seeks to minimize exposure to the real time wholesale electricity market while fulfilling flexible energy requests within a specific time horizon. The objective function of the aggregator can be expressed as follows:

$$
\min _{\phi_{t}\left(x_{t}\right)} \mathbb{E}\left[\sum_{t=1}^{N} \lambda_{t}\left(\phi_{t}\left(x_{t}\right)-s_{t}\right)^{+} \Delta t+\rho r_{N}\right]
$$

where $\phi_{t}\left(x_{t}\right)$ if the optimal policy, a function which maps the current state of the system to the amount of power supplied to the load at period $t$. The argument $x_{t}$ describes the state of the system at period $t$. The state space consists of three elements. The first element is the wholesale electricity price at period $t, \lambda_{t}$, the second element is the amount of available renewable energy at period $t, s_{t}$, and the third element is the amount of remaining energy demand at period $t, r_{t}$. Each time step has a step size $\Delta t$ and the entire horizon has $N$ time steps. In the last period, the aggregator incurs a penalty $\rho$ for each unit of energy that remains unfulfilled. The initial amount of energy demand is $R$. The aggregator must respect a capacity constraint which limits the rate at which loads can absorb power, $\phi_{t}\left(x_{t}\right) \leq C_{p}$. The aggregator must also respect a limit on the amount of power that can be procured from the real time electricity market, which is expressed as $\phi_{t}\left(x_{t}\right)-s_{t} \leq C_{m}$. The latter constraint could be integrated in the proposed coupling contract. In that case, the system operator could compensate the aggregator with a capacity payment for agreeing to adhere to a lower value of $C_{m}$. Nevertheless, our primary interest is to examine the degree to which demand flexibility is adequate in absorbing the adverse impacts of renewable power fluctuations, and therefore we focus on $C_{m}$ as a model parameter and study the sensitivity of our results to this parameter.

The remaining energy demand for flexible consumers evolves according to

$$
r_{t+1}=r_{t}-\phi_{t}\left(x_{t}\right) \Delta t
$$

The real time market price is determined from the optimal solution of a network model which is solved repeatedly over a short time horizon in the balancing market. Rather than adopting this analytical approach to model market prices, we treat it as a lognormal stochastic process and focus on extracting the parameters of the process from historical data, in order to apply a dynamic programming algorithm. Similarly, we assume that the wind power supply process obeys a Weibull distribution.

The renewable supply and real time price are driven by an underlying two-dimensional noise process that evolves on a trinomial recombinant lattice. The dynamics of the noise process are described by the following equations:

$$
\begin{aligned}
& n_{t+1}^{s, j}= \begin{cases}n_{t}^{s}+\sigma^{s} \sqrt{\frac{3}{2}} \sqrt{\Delta t}, & j=1 \\
n_{t}^{s}, & j=2 \\
n_{t}^{s}-\sigma^{s} \sqrt{\frac{3}{2}} \sqrt{\Delta t}, & j=3\end{cases} \\
& n_{t+1}^{\lambda, j}= \begin{cases}n_{t}^{\lambda}+\left(\sqrt{3} \rho+\sqrt{1-\rho^{2}}\right) \sigma^{\lambda} \sqrt{\frac{\Delta t}{2}}, & j=1 \\
n_{t}^{\lambda}-\sigma^{\lambda} \sqrt{1-\rho^{2}} \frac{2}{\sqrt{2}} \sqrt{\Delta t}, & j=2 \\
n_{t}^{\lambda}-\left(\sqrt{3} \rho-\sqrt{1-\rho^{2}}\right) \sigma^{\lambda} \sqrt{\frac{\Delta t}{2}}, & j=3,\end{cases}
\end{aligned}
$$

where $n_{t+1}^{s, j}$ is the value of the power supply process noise in state $j$ and period $t+1, n_{t}^{s}$ is the value of the noise process at period $t$ and the coefficient $\sigma^{s}$ controls the variance of the process. The notation is analogous for the underlying noise of the price process, $n_{t}^{\lambda}$. The coefficient $\rho$ controls the degree of correlation between the two processes. The transition probabilities from the current state $\left(n_{t}^{s}, n_{t}^{\lambda}\right)$ to the next state $\left(n_{t+1}^{s, j}, n_{t+1}^{\lambda, j}\right)$ for each $j \in\{1,2,3\}$ depend on the current state and are described in [10].

The noise processes generate the renewable supply and spot price processes by using the following transformations:

$$
\begin{aligned}
s_{t} & =\mu_{t}^{s} \lambda \exp \left(k^{-1} \log \log \left(1-N\left(n_{t}^{s}\right)\right)\right) \\
\lambda_{t} & =\mu_{t}^{\lambda} \exp \left(n_{t}^{\lambda}\right),
\end{aligned}
$$

where $N$ is the cumulative distribution function of the standard Gaussian distribution, and $k, \lambda$ are the parameters of the Weibull distribution. Consequently the resulting process $s_{t}$ approximately obeys a Weibull distribution, and the price process $\lambda_{t}$ approximately obeys a lognormal distribution.

The advantage of using a trinomial recombinant lattice lies in the fact that the state space of the optimal control problem exhibits quadratic growth in the length of the horizon, $N$, instead of exponential growth. Thus we can use a dynamic programming algorithm to solve the problem within a reasonable amount of computation time. Our approach follows the methodology which is outlined by Deng and Oren in [11], [10].

\section{RESULTS}

In this section we compare the performance of the optimal policy to alternative charging policies, and we examine the sensitivity of our results to various problem parameters. The results are sourced entirely from [12].

We focus our analysis on wind power supply. We use data from the western wind data set of the National Renewable Energy Laboratory for 2006 to calibrate the parameters of the wind power supply model of Eq. 3. The locations of the wind generator sites were selected in order to correspond to an integration target of $14,143 \mathrm{MW}$, according to data that is available in the California interconnection queue [13] and the California ISO renewable integration report [1]. We consider a time horizon of one day with hourly increments. We assume that loads place a request for $R=80,000 \mathrm{MWh}$, with a capacity constraint of $C_{p}=15,000 \mathrm{MW}$ on the rate at which they can absorb power. If we assume that a typical electric vehicle has a power rating of $3.6 \mathrm{~kW}$ and a mileage 
of $0.25 \mathrm{kWh}$ per mile, the demand model roughly represents the electricity demand of 4.167 million electric vehicles which travel 96 miles per vehicle per day. The aggregator respects a limit of $C_{m}=2,000 \mathrm{MW}$ on the rate of power that it can procure in the wholesale spot market. We have chosen $C_{m}$ to be a small fraction of $C_{p}$ in order to test our intuition that deferrable energy requests couple well with renewable power supply over an extended time horizon. If the coupled system were required to resort primarily to the real time market in order to perform adequately, then there is little reason to consider coupling contracts as a means of utilizing demand flexibility to absorb renewable supply fluctuations. The cost of unserved energy in the baseline is $\rho=5,000 \$ / \mathrm{MWh}$. This value is selected as an estimate of the average cost of not serving flexible energy requests for vehicle charging.

We compare the optimal control policy to a clairvoyant policy which perfectly forecasts wind power supply and spot market prices in advance, a naive charging policy which serves loads at maximum rate immediately after they place their request, and a model predictive controller (MPC) which implements the optimal control at each time step assuming that the price and wind processes will obey their unperturbed dynamics for the remaining horizon. The clairvoyant policy represents a bound on the potential benefits of forecasting. We find that, compared to the clairvoyant policy, the spot market expenditures of the optimal control policy are $36.3 \%$ higher. The MPC policy expends $41.4 \%$ more than the clairvoyant policy in the spot market, however, the MPC policy results in significant unserved load losses. The naive policy serves load to the greatest possible extent, however it expends $143.4 \%$ more than the clairvoyant policy in the spot market. The total cost of the dynamic programming algorithm, which is equal to real time market expenditures plus the cost of unserved load, is $24.8 \%$ higher than the total cost of the clairvoyant policy. The MPC policy costs are $139.9 \%$ higher than the costs of the clairvoyant policy, and the costs of the naive policy are $94.2 \%$ higher than the clairvoyant policy. Although model predictive control performs better than the naive policy in terms of realtime market expenditures, overall it performs worse than the naive policy due to the fact that it incurs high penalties for unserved energy. The results suggest that there is an inherently high cost of uncertainty in the problem.

We also perform a sensitivity analysis on the degree to which the aggregator can resort to the real time market and the cost of not serving demand. In particular, we vary $C_{m}$ around its baseline value from $C_{m}=1,000 \mathrm{MW}$ to $C_{m}=3,000$ MW. We find that limiting $C_{m}$ to $1,000 \mathrm{MW}$ results in lost load due to the possibility of highly adverse wind production outcomes, while as we increase $C_{m}$ the dynamic programming policy significantly outperforms the naive charging policy as the naive charging policy rapidly charges in the early periods and sheds excess amounts of available wind in the subsequent periods. Interestingly, the relative performance of the dynamic programming policy does not deteriorate compared to the clairvoyant policy as $C_{m}$ increases, hence there is a threshold beyond which increased spot market participation offers no added benefits to the aggregator. The penalty of unserved load is varied about its baseline value from $\rho=1,000 \$ / \mathrm{MWh}$ to $\rho=10,000 \$ / \mathrm{MWh}$. We observe that for low values of $\rho$ under sufficiently high real time prices, the dynamic programming policy may select to leave load unserved instead of procuring electricity from the spot market at a high price.

\section{CONCLUSION}

We describe a contract which couples renewable energy resources to flexible consumers. The aggregator is exposed to dynamic pricing for the amount of residual demand that cannot be fulfilled from its renewable resource assets. We formulate the problem faced by the aggregator as a stochastic optimal control problem and describe a trinomial recombinant lattice model that can be used for modeling the renewable energy supply and market price processes. The trinomial recombinant lattice model limits the growth of the state space of the stochastic optimal control problem, which makes it practical to use a dynamic programming algorithm for deriving the optimal charging policy for the aggregator. In our results section we observe that due to the high degree of uncertainty in the problem, an optimal control policy can lead to significant improvements compared to a naive charging approach, whereas heuristics control methods such as MPC may underperform due to the high risk of not serving demand. We also find that there is a threshold beyond which the ability of the aggregator to participate in the spot market offers no additional benefits. We finally observe that below a certain penalty for unserved load and for sufficiently high spot market prices, it may become advantageous for the aggregator to leave load unserved instead of covering demand through spot market procurements.

\section{ACKNOWLEDGMENT}

This research was funded by NSF Grant IIP 0969016, the US Department of Energy through a grant administered by the Consortium for Electric Reliability Technology Solutions (CERTS), by the Siemens Corporation under the UC Berkeley CKI initiative and by the Federal Energy Regulatory Commission.

\section{REFERENCES}

[1] C. Loutan and D. Hawkins, "Integration of renewable resources: Transmission and operating issues and recommendations for integrating renewable resources on the California ISO-controlled grid," California Independent System Operator, Tech. Rep., November 2007.

[2] R. Zavadil, "2006 Minnesota wind integration study, volume I," Enernex Corporation, Tech. Rep., November 302006.

[3] R. Gross, P. Heptonstall, D. Anderson, T. Green, M. Leach, and J. Skea, "The cost and impacts of intermittency: An assesment on the evidence on the costs and impacts of intermittent generation on the British electricity network," UK Energy Research Center, Tech. Rep., March 2006.

[4] F. van Hulle, "Large scale integration of wind energy in the european power supply: Analysis, recommendations and issues," European Wind Energy Association, Tech. Rep., 2005.

[5] S. Borenstein, M. Jaske, and A. Rosenfeld, "Dynamic pricing, advanced metering and demand response in electricity markets," University of California Energy Institute, Tech. Rep., October 2002.

[6] E. Hirst and B. Kirby, "Load as a resource in providing ancillary services," Oak Ridge National Laboratory, Tech. Rep., 1999.

[7] B. J. Kirby, "Spinning reserve from responsive loads," Oak Ridge National Laboratory, Tech. Rep., March 2003.

[8] J. Eto, "Demand response spinning reserve demonstration," Lawrence Berkeley National Laboratory, Tech. Rep., May 2007. 
[9] E. Hirst and B. Kirby, "Ancillary-service details: Dynamic scheduling," Oak Ridge National Laboratory, Tech. Rep., January 1997.

[10] S.-J. Deng and S. S. Oren, Valuation of Electricity Generation Capacity. MPS-SIAM Series on Optimization, 2005, ch. 31, pp. 655-667.

[11] — - "Incorporating operational characteristics and start-up costs in option-based valuation of power capacity," Probability in the Engineering and Informational Sciences, vol. 17, pp. 155-181, 2003.

[12] A. Papavasiliou and S. S. Oren, "Integration of contracted renewable energy and spot market supply to serve flexible loads," in Proc. 18th World Congress of the International Federation of Automatic Control, Milano, Italy, Ausgust 2011.

[13] The California ISO controlled grid generation queue as of January 8, 2010. [Online]. Available: http://www.caiso.com/14e9/14e9dd da1ebf0.pdf

Anthony Papavasiliou is a PhD student in the department of Industrial Engineering and Operations Research at UC Berkeley. He received a Bachelors degree in Electrical and Computer Engineering from the National Technical University of Athens, Greece. Anthony has worked on energy projects at the Federal Energy Regulatory Commission, the Xerox Palo Alto Research Center and the Energy Economics and Environmental Modeling Laboratory at the National Technical University of Athens. Anthony has received the 2008-2009 Sustainable Products and Solutions Program Fellowship and he has received award funding from the UC Berkeley Center for Entrepreneurship and Technology, the Center for Information Technology Research in the Interest of Society and the Big Ideas at Berkeley program.

Shmuel S. Oren is Professor of Industrial Engineering and Operations Research at the University of California, Berkeley. He is the Berkeley site director of PSERC. He has published numerous articles on aspects of electricity market design and has been a consultant to various private and government organizations including the Brazilian regulatory commission, the Alberta Utility Board, the Public Utility Commission, the Polish system operator and the Public Utility Commission of Texas. He holds a Bachelors and Masters degree in Mechanical Engineering and Material Engineering from the Technion in Israel and he received a Masters and $\mathrm{PhD}$ degree in Engineering Economic Systems in 1972 from Stanford. Dr. Oren is a fellow of INFORMS, and a fellow of the IEEE. 